\title{
Corela
}

Cognition, représentation, langage

HS-25 | 2018

Les procédés implicites dans l'interface sémantiquepragmatique

\section{Voyage de l'implicite à la composition du sens}

\section{Élodie Oursel}

\section{(2) OpenEdition}

Journals

Édition électronique

URL : http://journals.openedition.org/corela/6491

DOI : $10.4000 /$ corela.6491

ISSN : 1638-573X

Éditeur

Cercle linguistique du Centre et de l'Ouest - CerLICO

Référence électronique

Élodie Oursel, «Voyage de l'implicite à la composition du sens », Corela [En ligne], HS-25 | 2018, mis en ligne le 12 juillet 2018, consulté le 01 mai 2019. URL : http://journals.openedition.org/corela/6491

DOI : 10.4000/corela.6491

Ce document a été généré automatiquement le 1 mai 2019.

\section{(c) (i) (3) (2)}

Corela - cognition, représentation, langage est mis à disposition selon les termes de la licence Creative Commons Attribution - Pas d'Utilisation Commerciale - Partage dans les Mêmes Conditions 4.0 International. 


\title{
Voyage de l'implicite à la composition du sens
}

\author{
Élodie Oursel
}

\section{Introduction}

1 Dans cet article, nous faisons le point sur les apports à la notion de sens qu'a permis un projet de recherche sur la gestion de l'intercompréhension. Nous commençons par y exposer l'état initial du projet, qui devait porter sur la compréhension de l'implicite par des locuteurs non natifs à l'oral. L'interdisciplinarité de ce projet (qui allie didactique, analyse des interactions et psycholinguistique) a engendré une remise en question de l'opérabilité de la notion d'implicite, ainsi qu'une réorientation du projet. Redéfini, il a conduit à une réflexion sur ce qu'est le sens en interaction. Après avoir abordé l'évolution $\mathrm{du}$ projet de recherche, nous explicitons le cadre conceptuel, la méthodologie et les catégories d'observables utilisés dans le projet définitif, avant de revenir sur la notion de sens.

\section{Interdisciplinarité et compatibilité des notions opératoires}

2 Au départ, notre projet de recherche en didactique des langues visait à comprendre la façon dont les locuteurs francophones non natifs parviennent à donner du sens à l'implicite à l'oral, à faire le point sur les difficultés auxquelles ils étaient confrontés, et à proposer des solutions pour l'enseignement. Pour ce faire, nous avons abordé les formes de l'implicite d'une part, et les processus cognitifs de la compréhension de l'oral d'autre part, à travers des lectures en linguistique (Kerbrat-Orecchioni 1977 et 1986, Sperber et Wilson 1989, Authier-Revuz 1995, Récanati 2004 [2007] entre autres), en pragmatique (Austin 1955 [1962/1975], Grice 1967 [1975/1989/1991], Ehlich et Rehbein 1972, Thomas 1983 et 1984, Searle et Vanderveken 1985) et en didactique (Bremer, Roberts, Vasseur et al . 1996, Carette 2001, Cornaire 1998, Flowerdrew et Miller 2005, Hérédia-Deprez 1986, 
Lhote 1995, Noyau et Porquier (dir.) 1984) pour voir les solutions déjà proposées. Ces lectures ne couvrant pas les recherches en psycholinguistique de la compréhension de l'oral, nous les avons complétées (Caron 1989 [2008], Le Ny 2005, etc.).

La plupart des travaux en didactique et en linguistique se répondaient tout à fait, avec des conceptions de l'implicite sinon communes, relativement similaires : le sens d'un énoncé est composé d'un sens littéral, sans connotations, sans apports du contexte, auquel il faut ajouter un sens implicite, inféré ou induit à partir du contexte. Certains travaux en linguistique (Roulet, Auchlin, Moeschler et al. 1985, Rastier 2006 par exemple) et en pragmatique donnaient un sens plus englobant à l'implicite, se plaçant dans une perspective plus seulement sémantique, mais sémiotique et située : les objets, les gestes, le temps participent à la construction du sens de la situation. Quant aux travaux en sciences cognitives, en particulier ceux portant sur la construction du sens (compréhension, interprétation), ils posaient la question en d'autres termes que l'opposition entre implicite et explicite. La compréhension d'un message verbal y est conçue comme un ensemble de processus complexes, où le sens n'est pas décodé, mais construit à partir d'un faisceau d'indices. Dans les lignes qui suivent, nous entrons un peu plus en détail dans ces processus.

\subsection{Les processus en jeu dans l'interprétation}

4 Trois grands modèles de la compréhension permettent de comprendre les processus en jeu dans l'interprétation d'une production verbale. Selon un premier modèle, dit " ascendant ", l'interlocuteur enregistre les suites de sons, les découpe et les organise en suites de mots, et les structure en suites de séquences et de paragraphes oraux. Dans ce cadre, il est aisé de considérer le « décodage » d'un sens explicite, auquel s'ajouterait un processus d'interprétation du sens implicite, par exemple avec un calcul interprétatif complémentaire (voir par ex. l'approche modulaire proposée par Fodor 1983 [1986]).

5 Un deuxième modèle, dit « descendant ", a émergé dans les années 1960 et 1970, venant apparemment contredire le premier. Selon les études qui en sont à l'origine, l'interlocuteur donne du sens aux unités verbales grâce au contexte (verbal et non verbal). Une étude de Pollack et Pickett (1964) nous informe que lorsque l'on extrait des mots entiers d'une conversation spontanée et qu'ils sont soumis à l'écoute de participants, seulement 47 \% d'entre eux sont correctement identifiés. Même lorsqu'ils sont extraits d'une lecture à vitesse normale, donc en production plus contrôlée, le résultat ne dépasse pas $55 \%$ : le matériau sonore que nous interprétons sans mal en contexte n'est plus traité de la même manière hors contexte. Il pose de sérieux problèmes d'identification et ensuite d'interprétation : l'accès lexical devient hasardeux.

Une deuxième étude, de Warren et Warren en 1970, montre que si l'on remplace un phonème $\mathrm{e}^{1}$ par un bruit (de toux ou autre), les sujets de l'expérience ont l'illusion d'entendre le phonème remplacé (en l'occurrence, le -s- central de legislature), et situent ailleurs que sur ce phonème le moment d'apparition du bruit. L'expérience a également été réalisée sur une syllabe entière, en l'occurrence sur le -gis- central, avec les mêmes résultats. Dans cet article, il est également fait rapport d'une expérience au cours de laquelle des sujets écoutent un stimulus en boucle, sans pause pendant plusieurs minutes (en l'occurrence, tress), et déclarent ensuite avoir entendu, outre le stimulus, des mots tels que stress, mais aussi dress, Joyce, floris, florist et purse ${ }^{2}$. Dans le même article enfin, les auteurs rapportent que les télégraphistes chevronnés écoutant du morse ne 
transcrivaient généralement pas les signaux qui constituaient un mot avant que six à douze mots de plus aient été entendus. Au contraire, lorsque les messages étaient chiffrés ou contenaient des cotes d'actions en bourse, les télégraphistes changeaient de stratégie et transcrivaient les signaux plus immédiatement. Ce travail demandant une plus grande concentration, les signaux étaient transmis et transcrits plus lentement. Actuellement, un parallèle avec les pratiques des transcripteurs de corpus peut facilement être établi.

Ces travaux, entre autres, entrent en résonnance avec les travaux de Bartlett (1932) et de Schank et Abelson (1977) sur les schémas et les attentes. Il n'y a pas de correspondance exacte et fiable entre le signal sonore objectif et sa perception et son analyse par l'interlocuteur. Celui-ci semble en fait anticiper et effectuer ensuite une analyse rapide et superficielle afin de vérifier ses hypothèses. Le travail des télégraphistes suggère également que cette vérification a lieu en boucle, jusqu'à ce qu'une certaine quantité de signaux, produisant probablement un sens global, ait été interprétée. Cela est d'une certaine façon confirmé par des travaux plus récents comme ceux de Reichler-Béguelin (1988) et de Berrendonner (1990) sur le passage d'un acte énonciatif en mémoire discursive.

Rumelhart propose en 1975 de considérer que ces deux processus ne sont pas concurrents ou opposés, mais qu'ils interagissent l'un avec l'autre : la situation et les sons entendus avant un temps $t$ sont utilisés pour créer et affiner des attentes sur la production verbale à venir et sur l'évolution de la situation; les évolutions dans la situation et les nouveaux sons produits viennent confirmer certaines hypothèses, en infirmer d'autres, et en même temps, ils créent ou affinent les attentes concernant la suite. On en est ainsi venus à envisager ces processus comme complémentaires et interactifs (Elman et McClelland 1984).

\subsection{Conséquences pour la notion d'implicite}

9 Accordant une grande importance aux processus descendants dans l'interprétation, nous considérons qu'ils ont un rôle puissant dans la construction du sens. L'interlocuteur s'appuie certes sur les signaux sonores et sur l'ensemble des autres types d'indices émergeant et perçus par ses sens (indices visuels, tactiles, olfactifs, temporels, etc.), mais aussi sur ses attentes au sujet de ces signaux, de la situation dans laquelle il se trouve, et de l'activité dans laquelle il est engagé. De plus, le niveau de la réalité auquel il se situe dans son interprétation, son humeur, ses émotions ou sa disposition influencent sa capacité de concentration et par voie de conséquence la qualité et la quantité d'indices qu'il repère et qu'il interprète. Ses hypothèses portent à la fois sur le sens à donner aux signaux qui peuvent apparaitre, et sur le sens a priori accordé aux signaux verbaux et non verbaux déjà interprétés: l'interlocuteur échaffaude dans l'incertitude et cherche confirmation dans l'émergent. Si l'on se place non pas du point de vue linguistique, de celui du matériau physique et enregistrable, ou de la langue, mais du point de vue de l'interlocuteur et du matériau cognitif, perçu et interprété, les données nous semblent beaucoup moins «transparentes »: qu'est-ce qu'un mot sinon l'interprétation qu'a effectué l'interlocuteur d'une information perçue et identifiée comme un mot, et comme «ce» mot? La part subjective de l'identification, du découpage et de la construction du sens ne peut pas être négligée, si nous nous plaçons dans cette perspective.

10 Nous sommes alors confrontée à plusieurs constats. Le premier: le matériau verbal n'apparait pas à l'interlocuteur tel qu'il est, mais tel que celui-ci le perçoit. Le deuxième : 
le travail interprétatif débute dès l'identification des sons et des mots. Le troisième : le matériau verbal ne véhicule pas de sens en lui-même, puisque l'interlocuteur effectue un travail interprétatif dès la perception. Le quatrième: dans une perspective où l'on s'intéresse d'abord au travail de l'interlocuteur, une approche constructiviste du sens s'impose $^{3}$; le sens n'est pas dans les mots, il est construit par l'interprétant via un ensemble de processus complexes d'interprétation.

Dans le cadre de notre étude - qui ne se place pas au niveau de la langue mais au niveau de la parole, qui ne porte pas sur le matériau linguistique mais sur la collaboration des partenaires d'une activité qui utilisent ce matériau pour agir, et qui s'inscrit clairement dans une approche constructiviste du sens - les constats mentionnés rendent trop faible la place de l'explicite pour que cette notion reste opératoire. Par voie de conséquence, celle d'implicite ne l'est plus non plus. L'opérabilité de ces notions n'est cependant pas remise en question dans l'absolu. C'est bien la spécificité des conditions, des objets d'étude et du cadrage disciplinaire, épistémologique et théorique qui nous conduisent à cette conclusion particulière.

Le projet de recherche de départ, qui visait à étudier la compréhension de l'implicite, n'était plus faisable dans ces conditions. Une réorientation du projet s'en est suivie : nos analyses ont finalement porté sur la gestion de l'intercompréhension. Ce faisant, nous sommes restée dans le cadre interdisciplinaire posé, avons maintenu l'étude des moyens mis en œuvre par l'interlocuteur pour collaborer à la réussite de la communication, et avons pu dégager ce qui peut faire l'objet d'une interprétation. Avec la réorientation de notre projet s'est affinée notre arrière-plan conceptuel et théorique. Nous en présenterons les grandes lignes dans la section suivante ${ }^{4}$.

\section{Une nouvelle conception de la compréhension... et du sens}

13 Notre conception de la compréhension, ou plutôt de l'interprétation, et du sens a été déterminée entre autres par la notion de communicabilité du sens, et par les courants de l'action située et de l'action conjointe.

\subsection{La communicabilité du sens}

En forgeant la notion de communicabilité du sens, le philosophe Jacques (1985) s'oppose à la conception de la communication selon laquelle l'interlocuteur reconstruit ce que l'énonciateur lui donne à comprendre en décodant un sens déjà là, présent dans la matérialité du discours. Il propose au contraire une conception de la communication où la production du locuteur est matérielle, mais où l'interlocuteur a accès à un ensemble de sens potentiels. Selon le psychologue des interactions Brassac, qui s'en inspire, le sens émerge dans l'interaction, par sélection d'un sens potentiel, par vérification et $\mathrm{si}$ nécessaire, par négociation avant que les partenaires n'atteignent un accord: la matérialité verbale ne véhicule pas de sens, elle constitue un faisceau d'indices que les interactants interprètent. Ils sélectionnent un sens potentiel, en fonction des hypothèses posées sur la base de ce qui a émergé avant, et de ce qui émerge dans le moment présent. 


\subsection{L'action située}

15 Le modèle descendant nous incite à ne pas oublier que l'utilisation du langage est située dans un contexte précis, et à prendre une posture non logocentrée. Le courant de l'action située formalise cela en replaçant le langage dans une situation et dans une activité : son unité d'analyse est "the activity of persons-acting in setting" (Lave 1988: 177). Selon ce courant, les individus, les actions et les pensées n'ont de sens que dans la situation dans laquelle ils se trouvent: Theureau (1992 [2004]), faisant référence aux analyses de Suchman (1987) sur les interactions homme-machine, écrit que les individus agissent «dans une boucle réciproque d'interprétation, utilisant les instructions [de la photocopieuse] pour faire sens de l'environnement, et l'environnement pour faire sens des instructions » (ibid. : 14). Les activités se structurent à partir de l'immédiateté de la situation : les schémas se modèlent et se déroulent (comme une bobine) à mesure que la situation se développe. Le sens est indissociable de la situation dans laquelle il émerge : «Le sens n'est pas de la signification déformée par le contexte : la signification est du sens appauvri car coupé de son contexte » (Rastier $2006: 3$. de la version en ligne).

\subsection{L'action conjointe}

Si tout énoncé, tout acte a un potentiel de sens, et que l'interlocuteur ne fait que choisir un sens potentiel, il n'y a intercompréhension et donc réussite de la communication que si l'interlocuteur a un retour du producteur sur son choix, et que les deux s'accordent. Ainsi, la communication en interaction relève de l'action conjointe : elle est composée d'actions individuelles et d'un travail commun de coordination et de co-construction. C'est pourquoi, dans l'analyse d'interactions, nous distinguons l'hypothèse interprétative de chaque interactant (le sens potentiel choisi, qui reste une hypothèse sur le sens de l'énoncé tant qu'elle n'a pas fait l'objet d'un accord), l'interprétation (le sens que chacun accorde à l'énoncé après atteinte de l'accord; sens personnel, mais évalué comme compatible avec l'interprétation de l'autre), et la valeur interactantielle, qui est ce que les partenaires ont co-construit, et qui a été déposé dans le pot commun de l'intersubjectivité. La valeur interactantielle d'un énoncé (ou d'un acte) est le constituant essentiel de l'intercompréhension. De plus, l'unité minimale telle qu'établie par Brassac (2000) pour l'analyse de l'intercompréhension est composée de trois actions coréalisées : la production d'un acte porteur d'un potentiel de sens, la proposition de sélection d'un sens, et la confirmation ou l'infirmation de la sélection. Ainsi, les notions satellites de l'intercompréhension et l'unité minimale d'analyse se conforment à l'idée qu'il s'agit d'une action conjointe.

De la même façon, la définition que nous proposons se distingue de celle communément admise selon laquelle l'intercompréhension serait l'ajustement unilatéral de l'interlocuteur au locuteur, le premier traitant la production verbale par un calcul sémantico-pragmatique inverse à celui opéré par le producteur : «l'intercompréhension est le résultat d'un processus collaboratif d'élaboration du sens par les interactants. Ils s'intercomprennent lorsqu'ils parviennent à un accord dont ils sont coresponsables et qui émerge dans l'interaction [...]. L'intercompréhension est un espace sémiotique, une zone d'accord intersubjective, qui émerge dans l'entre-deux conversationnel grâce à la négociation active des interactants » (Oursel $2013: 51-52$ ). 
18 Maintenant que les notions et l'arrière-plan théorique utiles à notre propos ont été exposés, nous allons aborder la méthodologie employée pour les analyses avant de présenter les catégories d'observables établies.

\section{Méthodologie}

Dans cette troisième section, nous présenterons le corpus sur lequel les analyses ont porté, puis la démarche d'analyse utilisée.

\subsection{Corpus d'analyse}

Les données ont été recueillies dans trois corpus d'enregistrements audio. Le premier a été réalisé dans le bureau d'une écrivain publique, le deuxième dans le bureau de remise des visas d'une préfecture de police, et le troisième dans des secrétariats et scolarités de français langue étrangère ${ }^{5}$. Ces corpus ont été recueillis parce que :

21 - nous cherchions des terrains où la réussite de la communication revêtait un enjeu important pour étudier la gestion de l'intercompréhension : ces sites administratifs, où une bonne qualité d'intercompréhension peut avoir un impact important sur la vie des usagers, se sont révélés relativement denses en négociations ;

22 - dans un objectif de recherche en didactique des langues étrangères, la dimension exolingue des situations de communication permettait à la fois d'éclairer les problèmes spécifiques à ce genre de communication et d'augmenter la densité des négociations.

D'un point de vue quantitatif, les corpus sont répartis comme suit :

\begin{tabular}{|l|l|l|l|l|l|}
\hline & \multicolumn{2}{l|l}{ Durée } & \multicolumn{2}{l|}{ Nombre d'interactions } \\
\hline Écrivain public & $06: 29: 55$ & & 11 & \\
\hline Préfecture de police & $01: 12: 50$ & & 17 & \\
\hline Secrétariats de FLE & $00: 27: 01$ & & 8 & \\
\hline & $08: 09: 46$ & & 36 & \\
\hline
\end{tabular}

Les analyses qui ont donné lieu aux résultats suivants ont été réalisées de manière systématique sur les transcriptions intégrales des plus de 8 heures d'enregistrements audio, soit environ 400 pages de transcriptions.

\subsection{Démarche d'analyse}

Avec ce corpus, notre objectif était d'établir les observables sur lesquels nous allions pouvoir travailler pour analyser la gestion de l'intercompréhension. Notre objectif n'était donc pas, à ce stade, de proposer une modélisation du sens. Celle-ci s'est imposée une fois l'établissement des catégories d'analyse effectué.

Nous avons tenté de prendre un point de vue émique sur la valeur interactantielle des productions : pour chacune, nous nous sommes appuyée sur la réaction de l'interlocuteur 
puis sur la réaction du producteur, et sur la réaction de l'interlocuteur à nouveau, pour poser une hypothèse sur sa valeur. Nous avons ainsi respecté l'unité minimale d'analyse de l'intercompréhension présentée plus haut.

D'un point de vue terminologique enfin, nous nous inspirons largement des unités proposées par Roulet (1981) et ensuite par Roulet, Auchlin et Moeschler (1985) : nous parlons d'interaction, de transaction ${ }^{6}$, d'échange, d'intervention, et d'acte signifiant ${ }^{7}$.

\section{Catégories d'observables}

La négociation du sens et la gestion de l'intercompréhension n'avaient pas encore donné lieu à une réflexion approfondie sur les observables sur lesquels s'appuyer dans les analyses. C'est pourquoi nous avons établi quatre catégories d'observables en fonction de leur relation avec, et donc de leur façon de renseigner sur, l'hypothèse interprétative du producteur.

- Certaines productions dévoilent des informations sur l'hypothèse interprétative de l'interlocuteur ;

- certaines productions informent le partenaire sur l'état de l'hypothèse interprétative ;

- d'autres encore constituent des réactions fondées sur elle, mais qui ne la décrivent pas d'une manière ou d'une autre ;

- d'autres enfin sont sans rapport sémantique avec l'hypothèse interprétative.

\subsection{Les réactions qui informent sur le contenu de l'hypothèse interprétative}

Le premier type de réaction est une formulation de l'hypothèse interprétative (c'est-àdire l'hypothèse que fait l'interactant sur le sens de ce qu'il interprète, cf. 2.3.). Cette formulation sert généralement un des deux objectifs : elle met en partage cette hypothèse avec le producteur, lui permettant de l'évaluer (et ce faisant, d'évaluer la qualité de l'intercompréhension), ou elle est produite à l'intérieur d'une question ou d'une demande et fait alors partie d'une demande d'aide, manifestant une insatisfaction, comme c'est le cas dans l'extrait ci-dessous :

(1) Tiré du corpus Écrivain public Shamina. ${ }^{8}$

Contestation d'une facture pour le remplacement d'une vitre.

\begin{tabular}{|l|l|}
\hline 01. Jacqueline & alors euh à l'occasion de travaux (lit) \\
\hline 02. & alors en plus \\
\hline 03. & alors ça a été cassé à quelle occasion \\
\hline 04. & parce que là de la manière dont il écrit \\
\hline 05. & à l'occasion des travaux de suppression d'ac- (lit) \\
\hline 06. & c'est l'entreprise qui l'a cassé \\
\hline 07. & ou : c'est vous \\
\hline
\end{tabular}




\begin{tabular}{|l|l|}
\hline 08. Shamina & euh parce que euh avec nous \\
\hline 09. & parce que il y a eu euh beaucoup de vent \\
\hline 10. Jacqueline & oui \\
\hline 11. Shamina & notre fenêtre ouvrir \\
\hline 12. & notre cet- tout de suite c- fermer \\
\hline 13. & ça a cassé petit peu \\
\hline
\end{tabular}

Jacqueline interprète les écrits du document que Shamina lui a apporté à lire. À mesure de sa lecture, elle se construit une image mentale de la situation dont il est question, de la procédure dans laquelle Shamina se trouve et, lorsqu'elle aura obtenu les réponses à toutes ses questions, elle saura également ce qui est attendu d'elle. Dans les réactions en gras, Jacqueline évoque le contenu de l'image mentale qu'elle s'est construite ; elle fournit à Shamina un ensemble d'indices pour l'aider à se représenter l'état de cette image et pour participer à l'améliorer. Shamina interprète cette réaction comme une question, comme une demande de précisions, et elle apporte ce qui lui semble y répondre.

En l'occurrence, le contenu problématique dans l'interprétation de Jacqueline est un détail factuel sur un événement de l'histoire. Ces types de réactions informent généralement sur les éléments qui composent le sens de l'acte signifiant encore en construction.

\subsection{Les réactions qui informent sur l'état de l'hypothèse interprétative}

32 Ces réactions sont parfois moins informatives du point de vue de la composition du sens, mais elles sont importantes pour la gestion de l'intercompréhension. Elles prennent tantôt la forme d'évaluations comme "je n'ai pas compris...» ou «je ne vois pas pourquoi... » parfois suivies du contenu évalué, tantôt d'indices sur l'état de l'hypothèse interprétative :

(2) Tiré du corpus Préfecture de police Hua.

Obtention du titre de séjour.

\begin{tabular}{|l|l|l|}
\hline 01. Yveline & vous avez acheté les timbres & Hypothèse interprétative \\
\hline 02. & hein & « hein » \\
\hline 03. Hua & ouais & Évaluation \\
\hline 04. & c'est bon & \\
\hline 05. & il est là & \\
\hline 06. Yveline & alors on va regarder (murmure) & \\
\hline
\end{tabular}




\begin{tabular}{|l|l|l|}
\hline 07. Hua & (pose les timbres sur le comptoir) & \\
\hline 08. & neuf à quinze euh & \\
\hline 09. & trois fois cinquante-cinq & Hypothèse interprétative \\
\hline 10. & c'est ça & Hypothèse interprétative \\
\hline 11. Yveline & voilà (ton bas) & Hypothèse interprétative \\
\hline 12. & c'est ça (ton bas) & «c'est ça » \\
\hline & & Évaluation \\
\hline
\end{tabular}

Les formes « hein » et «c'est ça / » indiquent au partenaire une insatisfaction de la part de l'interprétant vis-à-vis de son hypothèse. Ici, l'interprétant est parvenu à une hypothèse, il la juge relativement crédible (suffisamment pour avouer son incertitude en la formulant), mais il n'en est pas assez sûr pour s'épargner de la soumettre à la validation de l'autre.

Ces types de réaction n'apportent pas à la modélisation du sens en tant que telles, mais en tant qu'elles pointent sur la formulation de l'hypothèse qui les précède ou les suit.

\subsection{Les réactions fondées sur l'hypothèse interprétative}

Ce type de réaction est le plus fréquent. Dans ces cas, l'interprétant ne met pas en mots le contenu de son hypothèse interprétative, il poursuit l'activité en s'appuyant dessus. Ce type de réaction permet ainsi de faire progresser l'interaction (forward orientation), contrairement aux deux précédents qui marquent une pause dans la progression thématique (backward orientation, Vandergrift et Goh, 2012: 30-32). L'extrait suivant illustre cela :

(3) Tiré du corpus Préfecture de police Shawn.

Renseignement sur une procédure pour l'obtention de la carte de séjour.

\begin{tabular}{|c|c|c|}
\hline $\begin{array}{l}01 . \\
\text { Shawn }\end{array}$ & euh je suis un étudiant & \\
\hline 02. & $\begin{array}{l}\text { et j'ai fait déjà le (sil) } \\
\text { l'examen médical }\end{array}$ & \\
\hline $\begin{array}{l}03 . \\
\text { Yveline }\end{array}$ & oui & \\
\hline $\begin{array}{l}04 . \\
\text { Shawn }\end{array}$ & $\begin{array}{l}\text { mais j'ai oublié le } \\
\text { certificat qui prouve } \\
\text { que je l'ai fait }\end{array}$ & \\
\hline $\begin{array}{l}05 . \\
\text { Yveline }\end{array}$ & $\begin{array}{l}\text { ah bah je peux pas } \\
\text { donner la carte euh ça }\end{array}$ & $\begin{array}{l}\rightarrow \text { interprétation (à reconstruire par l'analyste) : Shawn } \\
\text { souhaite une carte mais il n'a pas le certificat nécessaire ; il }\end{array}$ \\
\hline
\end{tabular}




\begin{tabular}{|l|l|}
\hline $\begin{array}{l}06 . \\
\text { Shawn }\end{array}$ & n- oui \\
\hline 07. & je- je sais \\
\hline
\end{tabular}

En 05, Yveline a généré une hypothèse interprétative sur les actes 01,02 et 04 , dont elle semble a priori satisfaite (sinon, elle aurait demandé de l'aide ou des précisions). Elle semble avoir construit une hypothèse sur la situation de Shawn, sur les documents qu'il a, sur celui qu'il veut, sur la nature du problème qu'il décrit, et sur ce qu'il peut attendre d'elle. Elle semble suffisamment satisfaite de son hypothèse pour s'appuyer dessus pour poursuivre. Sa réaction en 05 ne porte pas sur son hypothèse interprétative de la situation, elle est fondée sur l'hypothèse interprétative mais répond à la demande interprétée. C'est en cela que ce type de réaction constitue une réaction « vers l'avant ».

Ce type de réactions nécessite un travail plus important de la part de l'analyste et conduit à des suppositions sur le contenu de l'hypothèse interprétative moins précises et moins sûres que les premiers types de réactions. Néanmoins, elles apportent un complément utile à la modélisation : l'hypothèse interprétative d'une production $\mathrm{P}$ contient des idées sur les réactions possibles et appropriées à $\mathrm{P}$ et sur les attentes du producteur de $\mathrm{P}$ au sujet de la forme et du fond de ces réactions9.

\subsection{Les réactions sans rapport thématique avec l'hypothèse interprétative}

Les réactions sans rapport thématique avec l'hypothèse interprétative constituent en général un changement de sujet dans l'interaction. L'extrait suivant en présente deux :

(4) Tiré du corpus Écrivain public Chellamani 01.

Déclaration d'un incendie de voiture à l'assurance par courrier.

\begin{tabular}{|c|c|c|}
\hline 01. Jacqueline & on va lui faire une petite enveloppe & \multirow{4}{*}{ Séquence $^{10} 1$ : préparation de l'enveloppe } \\
\hline 02. & (ouvre le tiroir) & \\
\hline 03. & (sort une enveloppe) & \\
\hline 04. & (ferme le tiroir) & \\
\hline 05. Chellamani & le docteur il vient euh après-midi & \multirow{2}{*}{ Séquence $2:$ bavardage } \\
\hline 06. & c'est pour ça & \\
\hline 07. Jacqueline & ouh là & \multirow{2}{*}{ Séquence 3 : préparation de l'enveloppe } \\
\hline 08. & ça colle (s'occupe de l'enveloppe) & \\
\hline
\end{tabular}

Globalement, ces réactions sans rapport thématique avec l'hypothèse interprétative de la production précédente surviennent lorsque la transaction en cours laisse la place, pour un moment, à une parenthèse, lorsqu'un élément devient soudain saillant ou lorsque la 
transaction précédente est achevée. Ce type de réaction apporte peu d'informations sur l'hypothèse de l'interprétant. Cependant, il est utile à la modélisation car il met en lumière une dimension de la gestion de l'interaction: on ne prend pas la parole à n'importe quel sujet à n'importe quel moment. L'interaction est organisée et structurée, et son organisation et sa structure se co-construisent dans l'émergence de l'interaction (les travaux de Sacks, Schegloff et Jefferson ont également porté sur cela, 1974 par exemple).

L'ensemble des productions verbales enregistrées ont été soumises à classification et chacune a trouvé sa place dans une des quatre catégories d'observables.

\section{Retour sur le sens}

41 Nous avons vu que certains types de réactions offraient davantage d'indices sur le contenu de l'hypothèse interprétative, d'autres sur le degré de satisfaction que ressent l'interprétant vis-à-vis d'elle, d'autres encore sur la direction que prend l'interaction ou l'activité, par exemple. Les quatre types de réaction nous aident à concevoir la nature des objets sur lesquels l'interprétation peut porter et les différentes dimensions du sens.

\subsection{Les objets d'interprétation}

42 Les quatre exemples que nous avons extraits jusqu'ici nous offrent déjà des informations utiles. Nous avons pu voir que les réactions donnent à voir plus ou moins précisément ce que contient l'hypothèse interprétative qui les a précédées. Ainsi, par exemple :

(2) Tiré du corpus Préfecture de police Hua.

Obtention du titre de séjour.

\begin{tabular}{|l|l|l|}
\hline 01. Yveline & vous avez acheté les timbres & Hypothèse interprétative \\
\hline 02. & hein & « hein » \\
\hline 03. Hua & ouais & Évaluation \\
\hline 04. & c'est bon & \\
\hline 05. & il est là & \\
\hline 06. Yveline & alors on va regarder (murmure) & \\
\hline 07. Hua & (pose les timbres sur le comptoir) & \\
\hline 08. & neuf à quinze euh & Hypothèse interprétative \\
\hline 09. & trois fois cinquante-cinq & Hypothèse interprétative \\
\hline 10. & c'est ça & Hypothèse interprétative \\
\hline 11. Yveline & voilà (ton bas) & c'est ça (ton bas) \\
\hline 12. & & \\
\hline
\end{tabular}




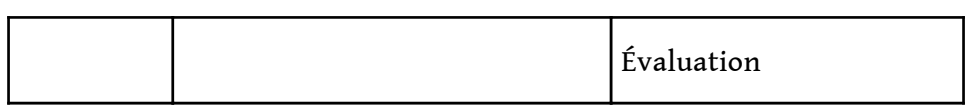

43 situation, plus précisément la supposition qu'une action antérieurement réalisée a pu, vraisemblablement, conduire l'usagère à la situation présente. Elle nous permet ainsi de penser que, dans une hypothèse interprétative, on peut trouver des idées d'actions passées déductibles de la situation présente.

Hua cherche les timbres dans son sac en même temps qu'elle réagit en 03 à 05 . Cela suggère qu'elle interprète la question d'Yveline sur l'achat passé aussi comme une requête de présentation des timbres. À travers cette réaction, acceptée par Yveline, on entrevoit une autre partie de l'hypothèse interprétative : Hua imagine quelles intentions Yveline avait en posant cette question, quels effets elle pouvait chercher à avoir, et réagit en accord avec la valeur illocutoire en sortant les timbres. En même temps, Hua manifeste une compréhension de l'organisation de l'interaction et de l'activité en cours : elle devait réaliser une action avant de venir, elle peut s'attendre à devoir montrer qu'elle a réalisé cette action. L'hypothèse interprétative porte donc aussi sur les intentions imaginables chez le producteur et sur les effets recherchés, ainsi que sur la structure de l'activité et de l'interaction.

En 08 et 09, Hua reprend un discours qu'elle a lu ou qui lui a été dit plus tôt dans la procédure : elle devait apporter neuf timbres à 15 euros et trois timbres à 55 euros. Pendant ces actes, elle laisse entendre le sens qu'elle donne aux objets qu'elle pose sur le comptoir : ce sont les timbres, qui correspondent a priori à la demande formulée plus tôt, dont la quantité et la valeur faciale sont celles attendues. En 10, elle demande confirmation, ce qu'Yveline s'empresse de lui donner en 11 et 12. Cet acte 10 est une manifestation du manque de certitude de Hua: elle espère que ses actions passées correspondent aux attentes, mais seule cette confirmation (et l'obtention du visa qui s'ensuit) suffiront à la rassurer complètement. Ici, on se situe au niveau du jugement de satisfaction que porte l'interprétante vis-à-vis de son interprétation, et c'est cela qui devient temporairement l'objet de l'interaction, et donc l'objet de l'hypothèse interprétative d'Yveline. En réagissant, Yveline répond bien sûr à la question, mais elle vient surtout rassurer Hua (le ton employé pour répondre réduit la distance et l'asymétrie relationnelles). Cette réaction suggère que dans l'hypothèse interprétative d'Yveline de l'acte 10, il y avait à la fois une question à laquelle Hua souhaitait une réponse (intentions et effets, types de réactions acceptables), et une demande de rassurance de la part d'une usagère inquiète et voulant bien faire (positionnement de l'usagère par rapport à l'agent, mise en mots d'émotions, demande de construction d'un lien social de bienveillance et de rassurance).

L'analyse de cet extrait a permis de voir que lorsque l'interlocuteur construit une hypothèse interprétative, il réalise un certain nombre de processus : il

- reconstitue par la logique le cours des actions qui a pu conduire à la situation de l'énonciation, et à celle évoquée dans l'interaction, si les deux situations sont différentes ;

- envisage les intentions et les effets recherchés par le producteur ;

- identifie l'organisation de l'activité et de l'interaction ;

- donne un sens aux objets de l'environnement ;

- lie une désignation à un objet ;

Corela, HS-25 | 2018 
- identifie les types de réactions acceptables et envisageables ;

- interprète la relation sociale que l'autre cherche à construire.

Les autres extraits offrent des indices complémentaires sur ce qui constitue une hypothèse interprétative : l'interlocuteur

- conçoit des liens logiques entre des éléments d'une situation ([3] «j'ai oublié le certificat qui prouve que je l'ai fait » $\rightarrow$ « ah bah je peux pas vous donner la carte »);

- reconstitue des circonstances ([1] « ça a été cassé à quelle occasion ", «c'est l'entreprise qui l'a cassé ou : c'est vous »);

- interprète s'il a l'« espace » verbal pour intervenir, pour bavarder, ou pour simplement apporter un retour minimal ([4] « le docteur il vient euh après-midi », [1] « oui »).

D'autres extraits seront nécessaires pour voir encore d'autres processus menés par l'interprétant pour construire son hypothèse interprétative : il

- identifie la nature des objets de l'environnement ;

(5) Tiré de Écrivain public Chellamani 02.

Complétion d'un formulaire de déclaration d'accident pour l'assurance (voiture brûlée)

\begin{tabular}{|l|l|}
\hline 01. Jacqueline & alors donc on va faire une photocopie \\
\hline 02. & donc ça photocopie \\
\hline 03. Chellakani & ça original \\
\hline 04. Jacqueline & voilà \\
\hline 05. Chellamani & original c'est c'est original \\
\hline 06. & c'est comme ça \\
\hline 07. Chellakani & ça ça origin- da- euh da: \\
\hline 08. Jacqueline & euh oui oui oui \\
\hline 09. & leuh duplicata \\
\hline 10. & mais c'est original \\
\hline 11. & oui \\
\hline 12. Chellakani & oui \\
\hline 13. & original \\
\hline 14. & oui \\
\hline 15. Chellamani & d'accord \\
\hline
\end{tabular}

49 - identifie un point de référence, « situe » la référence des propos ; 
(6) Tiré de Écrivain public Chellamani 02.

Lecture et appropriation d'un courrier de la C.P.A.M. ${ }^{11}$ au sujet de l'aide à la couverture médicale.

\begin{tabular}{|l|l|}
\hline 01. Jacqueline & vous devez remettre à l'organisme qui gère le contrat les attestations (lit) \\
\hline 02. & cette aide financière viendra en déduction de vos prochaines cotisations (lit) \\
\hline 03. & vous avez six mois (lit) \\
\hline 04. & donc jusqu'à fin juin \\
\hline 05. & pour choisir \\
\hline 06. & hein \\
\hline 07. Chellamani & sept cent euros \\
\hline 08. & c'est ça \\
\hline 09. Jacqueline & pour choisir \\
\hline 10. & voilà \\
\hline
\end{tabular}

50 - lie une information à une catégorisation et à un niveau de la réalité (niveau émotionnel, juridique, logique, etc.) ;

(7) Tiré de Écrivain public Anicham

Complétion d'une demande d'allocations familiales

\begin{tabular}{|l|l|}
\hline 01. Jacqueline & ah alors c'est pa :s par mois qu'il est payé \\
\hline 02. Anicham & non \\
\hline 03. & c'est pas par mois \\
\hline 04. & à tout à- à douze mois pour les euh je (sil) comptais pour la impôt \\
\hline 05. & c'est tout \\
\hline 06. Jacqueline & d'accord \\
\hline 07. & donc en fait euh \\
\hline 08. & ah oui \\
\hline 09. & il est \\
\hline 10. & (sil) \\
\hline 11. & il est salarié quand même \\
\hline
\end{tabular}




\begin{tabular}{|c|c|}
\hline 12. Anicham & euh oui \\
\hline 13. & pas salarié \\
\hline 14. Jacqueline & ah \\
\hline 15. & alors d'accord \\
\hline 16. & c'est pas salarié \\
\hline 17. & il touche quelque chose \\
\hline 18. & mais euh alors attendez \\
\hline 19. & je : c'est pas ça alors \\
\hline 20. & il est travailleur in- euh qu'est-ce qu'il fait exactement \\
\hline 21. Anicham & retou- retouches là \\
\hline 22. Jacqueline & euh \\
\hline 23. Anicham & pour les retousses (lapsus) \\
\hline 24. & retouches euh pour les \\
\hline 25. Jacqueline & ah \\
\hline 26. & il est artisan \\
\hline 27. Anicham & artisan \\
\hline 28. & oui \\
\hline 29. & voil- \\
\hline 30. Jacqueline & à son compte \\
\hline 31. Anicham & oui \\
\hline 32. Jacqueline & Ah d'accord d'accord \\
\hline
\end{tabular}

51 - juge la crédibilité des propos de l'autre, et la confiance qu'il lui accorde. ${ }^{12}$

(8) Tiré de Préfecture de police Dewei.

Obtention du titre de séjour.

\begin{tabular}{|l|l|}
\hline 01. Kader & des fois on : on casse le dos comme ça \\
\hline 02. Dewei & c'est pour ça \\
\hline 03. Kader & à chercher les dossiers \\
\hline
\end{tabular}




\begin{tabular}{|l|l|}
\hline 04. Dewei & ah bon (rire) \\
\hline 05. Kader & bah c'est lourd \\
\hline
\end{tabular}

52 À chaque processus réalisé par l'interprétant correspond la construction d'une partie de l'hypothèse interprétative: lorsqu'il envisage les intentions et les effets recherchés, il construit une représentation sur la direction de l'interaction et de la relation entre le producteur et lui; lorsqu'il identifie la fonction et la nature des objets de l'environnement, il construit une représentation sur les objets de la situation ; lorsqu'il lie une désignation à un objet, il identifie l'objet dans la réalité auquel il est fait référence et l'intègre dans sa représentation de ce sur quoi porte l'interaction, et ainsi de suite. On constate la complexité de l'hypothèse interprétative, et logiquement, lorsqu'elle a été validée, de l'interprétation. Mais nous pouvons organiser ses constituants en trois grands ensembles, que nous envisageons comme des dimensions, comme trois faces d'un prisme, contigües et indissociables.

\subsection{La composition du sens}

Les trois dimensions que nous proposons ici sont une dimension situante, une dimension structurante et une dimension relationnelle du sens des actes interprétés.

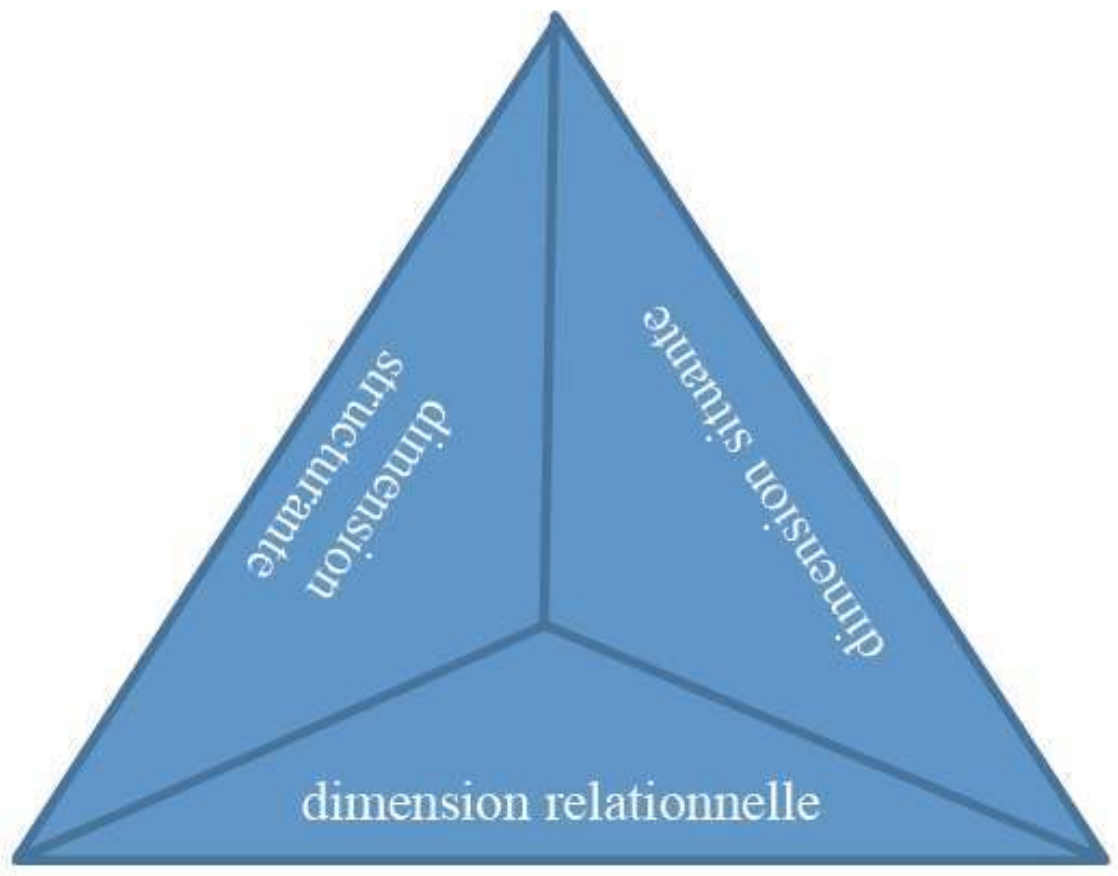

Chaque acte qui fait l'objet d'une interprétation est passé au crible de ces trois dimensions : quelle relation le producteur envisage-t-il entre nous ? Comment son acte se lie-t-il à ce qui précède dans l'activité et dans l'interaction, et quelle direction souhaite-til qu'elles prennent? De quoi est-il question, quel est l'objet de l'activité, quelle est la situation que je dois reconstituer ? $^{13}$

\subsubsection{La dimension situante}



sélectionne ce sur quoi porte l'acte, il lie les éléments verbaux aux réalités désignées, il construit une relation entre les éléments interprétés et l'environnement de l'interaction, il situe les agents (humains et non humains, animés ou non), il conçoit des liens logiques entre les éléments de la situation décrite et entre les éléments de la situation dans laquelle l'acte a émergé, il reconstitue des circonstances, conçoit l'organisation de l'environnement, ou catégorise une information.

57 La dimension situante construit une relation référentielle entre les objets interprétés et un environnement, une situation, une activité, etc. et une relation sémiologique entre les objets, l'interprétant et l'environnement. Le sens est ainsi incarné et situé.

\subsubsection{La dimension structurante}

Dans la construction de la dimension structurante du sens d'un acte, l'interprétant lie les actes à la progression et à l'organisation de l'échange, de l'action, de l'activité ou de l'interaction. L'interprétant juge par exemple de la complétude monologique d'une intervention et de la complétude dialogique d'un échange ; il interprète une intervention comme établissant l'ouverture ou la clôture d'un échange ou d'une transaction. Il identifie ainsi quelle direction le producteur de l'acte a choisi de donner à l'activité en agissant comme il l'a fait. Il considère également les intentions et les effets recherchés par le producteur de l'acte. La dimension structurante du sens concerne donc à la fois la direction que prend l'activité par l'acte interprété, et les directions possibles que cet acte permet, parmi lesquelles l'interprétant va faire un choix qui va impacter la poursuite de l'activité.

La dimension structurante de l'interprétation concerne donc l'interprétation de la place des éléments déjà produits, déjà là, dans la structure plus globale d'une activité et d'une interaction ainsi que la construction d'attentes quant à ce qui doit venir, quant aux réactions que l'interprétant peut avoir, et quant aux sens potentiels que ces réactions pourraient prendre.

\subsubsection{La dimension relationnelle}

60 La dimension relationnelle lie les différents indices perçus pour évaluer la relation entre les interactants, entre eux et les objets, entre eux et l'environnement, etc. Les relations peuvent être affectives, hiérarchiques, institutionnelles, générationnelles, psychologiques, etc. Si l'interaction présente des manifestations de l'évolution de la relation, l'interprétant identifie la direction qu'elle prend. La relation est également conditionnée par les rôles, les statuts et les places de chaque participant: la dimension relationnelle du sens concerne la place et l'image de chacun dans l'interaction. Le sens donné aux indices liés à la gestion des faces des participants est au cœur de cette dimension.

61 Ces trois dimensions sont complémentaires dans l'interprétation de la situation de communication: elles permettent de produire une hypothèse interprétative, puis de parvenir à une valeur interactantielle globale intégrant les différents aspects de l'interaction et de la situation. Mais certaines dimensions peuvent être plus prégnantes que d'autres à certains moments. Les dimensions peuvent prendre davantage d'importance en fonction du thème de la conversation, de la formulation employée, de 
l'identité et de la personnalité de l'interprétant, de son humeur, de son état d'esprit, de sa disposition, à un moment et en un lieu donnés. Des facteurs externes et des facteurs internes à l'interprétant peuvent donc influencer le poids qu'il accorde à chaque dimension dans la construction de son hypothèse.

\subsection{Discussion}

62 La modélisation du sens soumise ici peut rappeler des propositions existantes. On y retrouve en effet la notion de référence (dimension situante), omniprésente dans l'analyse du sens en sémantique, ou encore la valeur illocutoire et la force perlocutoire (dimension structurante), au cœur des analyses pragmatiques du discours. On y retrouve également la relation entre le locuteur et l'interlocuteur (dimension relationnelle), déjà présente dans le schéma des fonctions de la communication de Jakobson. Les dimensions du sens d'un acte sont construites à partir d'indices tels que le genre, le ton, le contexte, les intentions, etc. qu'Hymes avait déjà organisés dans son modèle des outils de la communication. Notre proposition n'est pas une rupture, elle s'inscrit dans une continuité avec les propositions passées. Cependant, elle nous semble s'en distinguer de trois manières.

Premièrement, la méthode par laquelle nous sommes parvenue à identifier ces trois dimensions du sens en interaction, via une analyse d'interactions dont l'objet était l'étude de la gestion de l'intercompréhension, permet de mettre en lumière d'une façon nouvelle ce qui est saillant dans la composition du sens.

Deuxièmement, de nombreux modèles portent sur la communication d'une manière ou d'une autre. La modélisation proposée ici ne porte pas sur la communication, ni sur les fonctions de la communication, ni sur la situation de communication, ni sur les outils de la communication. Elle porte sur le contenu de l'interprétation des participants à une interaction, interprétation d'un acte, verbal ou non verbal, réalisé dans le cadre d'une activité. Elle porte donc sur le sens que les participants donnent à ce qui émerge dans l'interaction. Mais ce qui émerge ne porte pas toujours et pas seulement sur le moment de l'énonciation : les partenaires font sens à la fois de ce dont il est question dans l'acte et de son retentissement dans la situation, dans l'activité, dans la relation, etc.

Troisièmement, notre proposition se concentre sur la composition minimale du sens, avec trois dimensions seulement, incitant à accorder une certaine place à chacune d'elles. Les analyses portent en effet le plus souvent sur la dimension situante du sens, particulièrement en didactique, où les activités de "compréhension de l'oral » visent surtout à reformuler l'objet de la conversation, et l'évolution de cet objet dans le cours de la conversation, délaissant les dimensions structurante et relationnelle ${ }^{14}$. On interrompt rarement un enregistrement pour demander aux apprenants le type de réaction que le locuteur attend de son partenaire à ce moment, ou encore comment la relation entre les interactants se manifeste ou évolue dans le cours de l'interaction. Une modélisation en trois dimensions peut inciter les analystes et les enseignants, à s'inquiéter de chacune d'elles un peu plus systématiquement dans l'analyse des situations de communication. 


\section{Conclusion}

66

Cet article a présenté les conséquences de l'interdisciplinarité de notre recherche sur notre conception de la composition du sens. De nombreux facteurs sont entrés en ligne de compte pour que nous arrivions à cette conclusion : en premier lieu l'incompatibilité des conceptions de la construction du sens dans le cadrage disciplinaire, épistémologique et théorique posé ; en deuxième lieu le corpus constitué d'interactions, qui fait émerger des négociations à chaque risque perçu de rupture de l'intercompréhension; en troisième lieu la taille du corpus, qui a permis de mettre en lumière des négociations sur les relations; en quatrième lieu le travail de classification des productions du corpus en catégories d'observables, qui a permis une lecture fine du déroulé des interactions.

La composition du sens en trois dimensions, situante, structurante et relationnelle, parait être applicable à toute situation de communication, même si elle est issue d'un corpus d'échanges en face-à-face et a été établie à partir de réactions et non de monologues ou de phrases fabriquées. Elle est également transférable à l'enseignement de la compréhension puisqu'elle permet de guider les apprenants de langue pour qu'ils envisagent tous les angles d'une interprétation.

\section{BIBLIOGRAPHIE}

AUSTIN J-L. (1955 conférences, publications 1962 [1970]), How to Do Things with Words, Cambridge (MA, US), Harvard University Press : Harvard Edition. Trad. française Quand dire c'est faire. Paris, Seuil.

AUTHIER-REVUZ J. (1995) Ces mots qui ne vont pas de soi. Boucles réflexives et non-coïncidences du dire. 2 tomes, Paris, Larousse : « Sciences du langage ».

BARTLETT F-C. (1932), Remembering : An Experimental and Social Study, Cambridge (GB), Cambridge University Press.

BERRENDONNER A. (1990), « Pour une macro-syntaxe », Travaux de linguistique, n²1, p. 25-36.

BRASSAC C. (2000), «Intercompréhension et communiaction », in BERTHOUD A-C. \& MONDADA

L. (dir.) Modèles du discours en confrontation, Berne (CH), Peter Lang, p. 219-228.

BREMER K., ROBERTS C. \& VASSEUR M-T. et al. (1996), Achieving Understanding. New York (NY, US), Longman.

CARETTE E. (2001), « Mieux apprendre à comprendre l'oral en langue étrangère », Le français dans le monde Recherches et applications 'Oral : variabilité et apprentissages', Paris, CLE International / CRAPEL.

CARON J. (1989 [2008]), Précis de psycholinguistique, Paris, Presses universitaires de France : «Quadrige».

CORNAIRE C. (1998), La compréhension orale, Paris, CLE International : Didactique des langues étrangères. 
EHLICH K. \& REHBEIN J. (1972), « Zur Konstitution pragmatischer Einheiten in einer Institution : Das Speiserestaurant », in WUNDERLICH D. (dir.), Linguistische Pragmatik, Frankfurt (DE), Athenaion, p. 209-254.

ELMAN J-L. \& MCCLELLAND J-L. (1984), « Speech perception as a cognitive process : The interactive activation model », in Speech and Language : Advances in Basic Research and Practice, $\mathrm{n}$ $\circ 10$, p. 337-373.

FLOWERDREW J. \& MILLER L. (2005), Second Language Listening. Theory and Practice, Cambridge (GB), New York (NY, US), Cambridge University Press : Cambridge Language Education.

FODOR J. (1983 [1986]), The Modularity of Mind. An Essay on Faculty Psychology, Cambridge (MA, US), MIT Press. Trad. fr. : La Modularité de l'esprit : essai sur la psychologie des facultés, Paris, Minuit : Propositions.

GRICE H-P. (1967 conférences, publication 1975 [1989]), « Logic and conversation », in COLE P. \& MORGAN J-L. (eds.), Syntax and semantics, vol. 3. New York : Academic Press, p. 41-58. Réimprimé dans Studies in the Way of Words. Cambridge (MA, US), Londres (GB), Harvard University Press, p. $22-40$.

HÉRÉDIA-DEPREZ C. de (1986), « Intercompréhension et malentendus. Étude d'interactions entre étrangers et autochtones ", Langue française, $n^{\circ} 71$, p. 48-69.

HYMES D-H. (1972), « On communicative competence », in PRIDE J-B. \& HOLMES J. (eds.) Sociolinguistics, Harmondsworth (GB), Penguin, p. 269-293.

HYMES D-H. (1984), Vers la compétence de communication, Paris, Hatier-Didier : Langues et Apprentissage des Langues.

JACQUES F. (1985), L'espace logique de l'interlocution, Paris, Presses universitaires de France.

KERBRAT-ORECCHIONI C. (1977), La connotation, Lyon, Presses universitaires de Lyon.

KERBRAT-ORECCHIONI C. (1986), L'implicite, Paris, Armand Colin.

LAVE J. (1988), Cognition in Practice : Mind, Mathematics, and Culture in Everyday Life, Cambridge (GB), Cambridge University Press.

LE NY J-F. (2005), Comment l'esprit produit du sens : Notions et résultats des sciences cognitives, Paris, Odile Jacob.

LHOTE É. (1995), Enseigner l'oral en interaction : percevoir, écouter, comprendre, Paris, Hachette.

POLLACK I. \& PICKETT J-M. (1969), “Intelligibility of excerpts from fluent speech : auditory vs structural context”, Journal of Verbal Learning and Verbal Behaviour, n 3, p. 79-84.

NOYAU C. \& PORQUIER R. (dir.) (1984), Communiquer dans la langue de l'autre, Paris, Presses universitaires de Vincennes, p. 130-152.

OURSEL É. (2013), Des interactions de service entre francophones natifs et non natifs. Analyse de la gestion de l'intercompréhension et perspectives didactiques, Thèse de doctorat co-dirigée par Sophie Moirand et Richard Duda, Université Paris 3 - Sorbonne nouvelle.

RASTIER F. (2006), « De la signification lexicale au sens textuel : éléments pour une approche unifiée », Texto !, $\mathrm{n}^{\circ} \mathrm{XI} / 1$ en ligne. Disponible à l'adresse :

http://www.revue-texto.net/Inedits/Rastier/Rastier_Signification-lexicale.html (consulté le 04/03/2016).

RECANATI F. (2004 [2007]), Literal Meaning, Cambridge, Cambridge University Press. Trad. fr. : Le sens littéral, langage, contexte, contenu, Paris, Edition de l'éclat. 
REICHLER-BÉGUELIN M-J. (1988), « Anaphore, cataphore et mémoire discursive », Pratiques, n57, p. 15-43.

ROULET E. (1981), «Échanges, interventions et actes de langage dans la structure de la conversation ", Études de linguistique appliquée, n 44, p. 7-40.

ROULET E., AUCHLIN A., MCESCHLER J. et al. (1985), L'articulation du discours en français contemporain, Berne $(\mathrm{CH})$, Peter Lang.

RUMELHART D-E. (1975), « Notes on a schema for stories », in BOBROW D-G. \& COLLINS A. (eds.), Representation and Understanding, New York (NY, US), Academic Press, p. 211-236.

SACKS H., SCHEGLOFF E-A. \& JEFFERSON G. (1974), « A simplest systematics for the organization of turn-taking for conversation », Language, $n^{\circ}$ 50/4, p. 696-735.

SCHANK R-C. \& ABELSON R-P. (1977), Scripts, Plans, Goals, and Understanding : An Inquiry into Human Knowledge Structures, Hillsdale (NJ, US), Lawrence Erlbaum.

SEARLE J-R. \& VANDERVEKEN D. (1985), Foundations of Illocutionary Logic, Cambridge (GB), Cambridge University Press.

SPERBER D. \& WILSON D. (1989), La pertinence, Communication et cognition, Paris, Éditions de Minuit.

SUCHMAN L. (1987 [2007]), Plans and Situated Actions : the Problem of Human/Machine Communication [Human-Machine Reconfigurations. Plans and Situated Actions], Cambridge (GB), Cambridge University Press.

THEUREAU J. (1992 [2004]), « L'hypothèse de la cognition (ou action) située et la tradition d'analyse du travail de l'ergonomie de langue française », @ctivités, nº 1/2, p. 11-25.

THOMAS J. (1983), “Cross-cultural pragmatic failure”, Applied Linguistics, nº 4, p. 91-112.

THOMAS J. (1984), « Cross-cultural discourse as 'unequal encounter' : Towards a pragmatic analysis ", Applied Linguistics, ${ }^{\circ}$ 5, p. 226-244.

VANDERGRIFT L. \& GOH C. (2012), Teaching and Learning Second Language Listening: Metacognition in Action, New York (NY, US), Routledge.

WARREN R-M. \& WARREN R-P. (1970), “Auditory illusions and confusions”, Scientific American, $\mathrm{n}$ - 223, p. 30-36.

\section{ANNEXES}

\section{ANNEXE 1}

Différents processus réalisés par l'interprétant avec des exemples tirés des extraits de l'article :

\begin{tabular}{|l|l|}
\hline $\begin{array}{l}\text { il donne un sens aux objets de } \\
\text { l'environnement, en identifiant leur nature et } \\
\text { une ou plusieurs de leurs fonctions }\end{array}$ & [5] «ça photocopie » «ça original » \\
\hline $\begin{array}{l}\text { il lie une désignation à un objet ou à un } \\
\text { concept }\end{array}$ & $\begin{array}{l}\text { [2] « vous avez acheté les timbres » } \rightarrow \text { (pose les } \\
\text { timbres surle comptoir) }\end{array}$ \\
\hline
\end{tabular}




\begin{tabular}{|c|c|}
\hline $\begin{array}{l}\text { il identifie un point de référence, «situe " la } \\
\text { référence des propos }\end{array}$ & [6] « vous avez six mois (lit) donc jusqu'à fin juin » \\
\hline $\begin{array}{l}\text { il conçoit des liens logiques entre les éléments } \\
\text { d'une situation }\end{array}$ & $\begin{array}{l}\text { [3] «j'ai oublié le certificat qui prouve que je l'ai } \\
\text { fait» } \rightarrow \text { «ah bah je peux pas vous donner la carte» }\end{array}$ \\
\hline il reconstitue des circonstances & $\begin{array}{l}\text { [1] "à l'occasion de travaux [...] ça a été cassé à } \\
\text { quelle occasion [...] c'est l'entreprise qui l'a cassé ou : } \\
\text { c'est vous" }\end{array}$ \\
\hline $\begin{array}{l}\text { il lie une information à une catégorisation et à } \\
\text { un niveau de la réalité }\end{array}$ & $\begin{array}{l}{[7] \text { "c'est pas par mois qu'il est payé }[. . .] \text { il est }} \\
\text { salarié quand même }[. . .] \text { c'est pas salarié }[. . .] \text { il est } \\
\text { travailleur in- }[. . .] \text { ah il est artisan }[. . .] \text { à son } \\
\text { compte" }\end{array}$ \\
\hline $\begin{array}{l}\text { il juge de la crédibilité des propos de l'autre } \\
\text { (et de la confiance qu'il leur accorde) }\end{array}$ & {$[8]$ «ah bon » } \\
\hline $\begin{array}{l}\text { il envisage les intentions et les effets } \\
\text { recherchés par le producteur d'un acte }\end{array}$ & $\begin{array}{l}\text { [3] «j'ai oublié le certificat qui prouve que je l'ai } \\
\text { fait » } \rightarrow \text { «ah bah je peux pas vous donner la carte» }\end{array}$ \\
\hline $\begin{array}{l}\text { il interprète la relation sociale que l'autre } \\
\text { chercherait à construire }\end{array}$ & $\begin{array}{l}{[2] \text { «c'est ça » } \rightarrow \text { «voilà c'est ça » }} \\
{[4] \text { «le docteur il vient euh après-midi }}\end{array}$ \\
\hline $\begin{array}{l}\text { il identifie l'organisation de l'activité et de } \\
\text { l'interaction, les types de réactions adéquats, } \\
\text { la direction de l'interaction }\end{array}$ & $\begin{array}{l}{[2] \text { «vous avez acheté les timbres» } \rightarrow \text { (pose les }} \\
\text { timbres sur le comptoir) }\end{array}$ \\
\hline il identifie l'« espace » verbal pour intervenir & $\begin{array}{l}{[4] \text { «le docteur il vient euh après-midi » }} \\
{[1] \text { « oui » }}\end{array}$ \\
\hline $\begin{array}{l}\text { il reconstitue par la logique le cours des } \\
\text { actions qui a pu conduire à la situation } \\
\text { évoquée et/ou à la situation présente }\end{array}$ & [2] « vous avez acheté les timbres » \\
\hline
\end{tabular}

\section{ANNEXE 2}

Exemple d'une brève analyse tridimensionnelle du sens de quelques actes interprétés

[4] « alors euh à l'occasion de travaux (lit) alors en plus alors ça a été cassé à quelle occasion parce que là de la manière dont il écrit à l'occasion des travaux de suppression d'ac- (lit) c'est l'entreprise qui l'a cassé ou c'est vous »

\section{Dimension situante du sens :}

Situation évoquée différente de la situation de communication.

Dans la situation évoquée, il est question de travaux réalisés dans des circonstances à éclaircir.

Dans la situation de communication, l'écrivain public prend connaissance d'un courrier à l'intention de l'usagère afin de se faire une idée du problème pour lequel elle sollicite cette transaction de service. 


\section{Dimension structurante :}

Au niveau de l'activité : la lecture du document fait partie de la phase d'exposition de la situation-problème de l'usagère et de construction de son dossier mental par l'agent. Cette étape implique que l'agent se fasse une idée claire de la situation afin de pouvoir évaluer l'objectif de la transaction.

Au niveau de l'intervention : l'agent utilise les indices fournis par le document pour concevoir la situation dans laquelle se trouve l'usagère dans les détails. Un détail sur les circonstances exactes du bras de vitre pose problème. Ce problème doit être résolu pour que l'agent puisse poursuivre sa construction du dossier mental et porter un regard professionnel sur la demande de l'usagère. Elle ouvre donc une question pour trouver l'information manquante. On peut supposer qu'elle espère et attend une réponse.

\section{Dimension relationnelle :}

Au niveau de l'activité : les interactantes ont une relation d'agent administratif et d'usagère, elles sont dans une relation de service administratif et agissent en conséquence, chacune réalisant les tâches qui lui sont dévolues.

Au niveau de l'intervention : l'agent a besoin d'une information, elle suppose que l'usagère détient cette information et entre dans une relation de demande d'aide. L'usagère est invitée à prendre une posture de facilitatrice dans l'interprétation de sa situation.

\section{NOTES}

1. Et les frontières des phonèmes précédent et suivant, qui pourraient fournir des indices quant au phonème central.

2. En français : boucle, stress, robe, Joyce, (invention), fleuriste, porte-monnaie.

3. C'est d'ailleurs pour respecter cette approche constructiviste du sens que nous avons délaissé le terme "compréhension" (en latin prendre quelque chose avec soi) et que nous lui préférons " interprétation » (du latin choisir un parmi plusieurs).

4. Pour un cadrage plus complet, se reporter à Oursel (2013).

5. La plupart des enregistrements du corpus Secrétariats de FLE ont été empruntés au groupe Fleuron: Documents Fleuron - Équipe Acquisition et apprentissage des langues (CRAPEL) U.M.R. 7118 - ATILF - C.N.R.S. Université Nancy 2.

6. Cette équipe a ensuite modifié ses unités de base, en particulier en abandonnant l'unité de la transaction, jugée redondante avec celle de l'échange. Du point de vue pragmatique, dans les interactions administratives, la transaction a une valeur tout à fait différente de l'échange : elle est l'unité de la production d'un service. C'est pourquoi nous avons préféré garder un découpage et une terminologie moins récents mais plus adaptés au corpus étudié.

7. Dans nos analyses, nous incluons les productions non verbales, les changements chez les objets, etc. Nous avons donc modifié le terme d'acte d'énonciation, utilisé par l'équipe, pour rendre compte de cette différence d'objet.

8. Conventions de transcription: la transcription est orthographique; à chaque pseudonyme correspond l'ouverture d'un tour de parole ou la production d'un retour minimal ; à chaque numéro correspond un acte signifiant (enregistré en mémoire discursive) ; les indications entre parenthèses concernent les objets, le paraverbal, le non verbal ou des commentaires de la transcriptrice; (sil) indique les silences de 0,5 seconde ou plus;: indique un allongement phonémique ; les soulignements indiquent un chevauchement entre deux productions ; - indique 
une interruption ou une troncation ; les majuscules indiquent une augmentation de l'intensité du signal sonore; le gras et les italiques sont utilisés pour faciliter les analyses en guidant le regard sur ce qui est étudié.

9. L'adéquation entre production et réaction a fait l'objet de nombreux travaux en analyse des interactions (travaux sur les paires adjacentes comme ceux de Sacks, Schegloff et Jefferson 1974) et en pragmatique (principe de coopération de Grice et autres règles du discours) entre autres. Ces travaux nous aident à mieux connaitre le fonctionnement de la compétence de communication (Hymes 1972 et 1984).

10. Nous utilisons ici le terme « séquence " pour désigner les différents types d'unités (échanges ou interventions) ouverts ou initiés au cours de l'extrait.

11. Caisse primaire d'assurance maladie.

12. L'annexe 1 récapitule les processus et les exemples présentés ici.

13. Un exemple d'analyse tridimensionnelle est proposé en annexe 2 pour une partie de l'extrait (4).

14. Au niveau des pratiques scientifiques, il semble que certains champs se soient spécialisés dans l'une ou l'autre dimension (la sémantique avec la dimension situante, l'analyse des conversations avec la dimension structurante, la microsociologie et l'interactionnisme symbolique pour la dimension relationnelle). Nous regrettons justement que l'ensemble des dimensions du sens ne soient pas considérées en même temps dans les analyses.

\section{RÉSUMÉS}

Cet article relate les détours d'un parcours de recherche doctorale sinueux. L'interdisciplinarité du projet dont il est question (didactique, analyse des interactions et psycholinguistique) a mis au jour une incompatibilité des conceptions du sens et de l'implicite. Le projet visait à étudier les moyens de communication dont dispose l'interlocuteur pour gérer l'intercompréhension. Afin de mener les analyses, quatre types d'observables (réactions de l'interlocuteur) ont été dégagés: celles qui informent directement sur le contenu de son interprétation (les reformulations par ex.) ou sur sa satisfaction vis-à-vis de cette interprétation ( je vois pas pourquoi »), celles qui changent de sujet (ouverture d'un nouveau topic), et celles qui poursuivent l'interaction «vers l'avant». Ces quatre types d'observables ont conduit à une conception tridimensionnelle du sens : une dimension situante du sens (permet de se représenter mentalement la scène, de situer l'objet du message) ; la dimension structurante du sens (porte sur l'organisation du discours et de l'interaction); la dimension relationnelle du sens (fait ressentir l'évolution de la relation entre les interactants). Cette modélisation remet en question la notion d'implicite ainsi que la distinction entre connotation et dénotation.

This article tells us about the twists and turns of a doctoral research. The interdisciplinarity of the project at stake (didactics, interactions analysis and psycholinguistics) showed an incompatibility in the conceptions of meaning and implicitness. The project aimed to study the means the co-speaker has to deal with mutual understanding. To analyse them, four kinds of observable data (co-speaker's reactions) have been considered: those that let know about the actual content of the interpretation (such as rephrasing) or about the co-speaker's feeling towards it («ah, I see »), those that change topics and those that keep the conversation going forward. Uncovering those four types of observable data and analysing the reactions helped led 
to a tri-dimensional conception of meaning : a situating dimension (allows to visualize the scene, to situate the object of the message), a structuring dimension (shows the organization of the discourse and of the conversation), and a relational dimension (makes the co-speaker feel which way the relationship with the speaker is evolving). This model questions the notion of implicitness as well as the distinction between connotation and denotation.

INDEX

Keywords : mutual understanding, implicitness, interdisciplinarity, meaning, interaction Mots-clés : intercompréhension, implicite, interdisciplinarité, sens, interaction

\section{AUTEUR}

\section{ÉLODIE OURSEL}

Structures formelles du langage, U.M.R. 7023

Université Paris 8 - Vincennes Saint-Denis 